\title{
Apamin suppresses biliary fibrosis and activation of hepatic stellate cells
}

\author{
JUNG-YEON KIM ${ }^{1}$, HYUN-JIN AN ${ }^{1}$, WOON-HAE KIM ${ }^{1}$, YOON-YUB PARK ${ }^{2}$, \\ KYUNG DUCK PARK ${ }^{3}$ and KWAN-KYU PARK ${ }^{1}$ \\ Departments of ${ }^{1}$ Pathology, ${ }^{2}$ Physiology and ${ }^{3}$ Dermatology, College of Medicine, \\ Catholic University of Daegu, Daegu 705-718, Republic of Korea
}

Received August 23, 2016; Accepted March 7, 2017

DOI: $10.3892 /$ ijmm.2017.2922

\begin{abstract}
Cholestatic liver disease is characterized by the progressive destruction of biliary epithelial cells (BECs) followed by fibrosis, cirrhosis and liver failure. Activated hepatic stellate cells (HSCs) and portal fibroblasts are the major cellular effectors of enhanced collagen deposition in biliary fibrosis. Apamin, an 18 amino acid peptide neurotoxin found in apitoxin (bee venom), is known to block $\mathrm{Ca}^{2+}$-activated $\mathrm{K}^{+}$channels and prevent carbon tetrachloride-induced liver fibrosis. In the present study, we aimed to ascertain whether apamin inhibits biliary fibrosis and the proliferation of HSCs. Cholestatic liver fibrosis was established in mouse models with 3,5-diethoxycarbonyl-1,4-dihydrocollidine (DDC) feeding. Cellular assays were performed on HSC-T6 cells (rat immortalized HSCs). DDC feeding led to increased hepatic damage and proinflammtory cytokine levels. Notably, apamin treatment resulted in decreased liver injury and proinflammatory cytokine levels. Moreover, apamin suppressed the deposition of collagen, proliferation of BECs and expression of fibrogenic genes in the DDC-fed mice. In HSCs, apamin suppressed activation of HSCs by inhibiting the Smad signaling pathway. These data suggest that apamin may be a potential therapeutic target in cholestatic liver disease.
\end{abstract}

\section{Introduction}

Hepatic fibrosis is caused by various types of chronic liver injury. This progressive pathological process is described as accumulation of extracellular matrix (ECM) proteins in and around injured liver tissues (1). Cholestasis results in intrahepatic accumulation of cytotoxic bile acids and hepatic inflammation, which is then followed by biliary fibrosis,

Correspondence to: Dr Kwan-Kyu Park, Department of Pathology, College of Medicine, Catholic University of Daegu, 3056-6 Daemyung-4-Dong, Daegu 705-718, Republic of Korea E-mail: kkpark@cu.ac.kr

Key words: biliary fibrosis, hepatic stellate cells, apamin, anti-fibrotic effect cirrhosis and finally end-stage liver disease $(2,3)$. Cholestatic liver disease such as primary biliary cirrhosis and primary sclerosing cholangitis is characterized by a progressive destruction of biliary epithelial cells (BECs) and inflammatory and autoimmune disorders $(4,5)$.

Proliferating BECs have been shown to secrete transforming growth factor- $\beta 1$ (TGF- $\beta 1$ ) and platelet-derived growth factor (PDGF), which stimulate the activation and proliferation of hepatic stellate cells (HSCs) and portal fibroblasts (6,7). Activated HSCs and portal fibroblasts cause enhanced collagen deposition and are the major cellular effectors in liver fibrosis $(1,8)$. This ultimately leads to excessive generation of ECM and accelerates the progression of fibrosis (9). Thus, the suppression of proliferating BECs and activated HSCs has been considered to be a therapeutic target for treating liver fibrosis.

Apamin is an 18 amino acid peptide neurotoxin found in apitoxin (bee venom) (10). It has long been known as a specifically selective blocker of $\mathrm{Ca}^{2+}$-activated $\mathrm{K}^{+}(\mathrm{SK})$ channels (11). These channels play an important role in mediating the increase in transepithelial secretion due to increases in intracellular $\mathrm{Ca}^{2+}$ (12). Moreover, apamin has been demonstrated to exhibit anti-inflammatory and anti-fibrotic activity in various cell types and mouse models $(13,14)$. A previous study carried out by our group confirmed that apamin is an anti-fibrotic agent which acts through suppression of TGF- $\beta 1$ induced hepatocyte epithelial-mesenchymal transition (13). However, the effects of apamin in biliary cirrhosis and the molecular mechanism underlying HSC proliferation have not been explored.

In the present study, we fed mice with 3,5-diethoxycarbonyl-1,4-dihydrocollidine (DDC), which induces sclerosing cholangitis and biliary fibrosis. We demonstrated that apamin inhibited DDC-induced liver fibrosis and mediated BEC proliferation and ductular reaction, which are repair responses to cholestatic injury. Moreover, apamin treatment caused the suppression of activated HSCs through the TGF- $\beta 1 / \mathrm{Smad}$ signaling pathway.

\section{Materials and methods}

Reagents. Apamin was purchased from Sigma (St. Louis, MO, USA). TGF- $\beta 1$ was purchased from R\&D Systems 
(Minneapolis, MN, USA) and dissolved in $4 \mathrm{mM} \mathrm{HCl}$ containing $0.1 \%$ bovine serum albumin (BSA).

DDC-induced mouse model of biliary fibrosis. For induction of liver injury, 8-week-old C57BL/6 male mice (20-25 g; Samtako, Osan, Korea) were selected. Male C57BL/6 mice were fed a control diet or a DDC supplemented diet $(0.1 \%)$ for 4 weeks to induce advanced biliary fibrosis as previously described (15). All animal protocols were approved by the Institutional Animal Care and Use Committee of Catholic University of Daegu (Daegu, Korea). The mice received an intraperitoneal injection of apamin $(0.1 \mathrm{mg} / \mathrm{kg})$ dissolved in saline twice a week. Mice were sacrificed after 4 weeks from the first DDC diet administration.

Cell culture. HSC-T6 cells, an immortalized rat hepatic stellate cell line, which has a stable phenotype and biochemical characteristics, was kindly provided by Dr S.L. Friedman (Liver Center Laboratory, San Francisco General Hospital, San Francisco, CA, USA). Cells were cultured at $37^{\circ} \mathrm{C}$ in a humidified incubator under a $5 \% \mathrm{CO}_{2}$ atmosphere. HSC-T6 cells were seeded in complete medium for $24 \mathrm{~h}$. The cells were changed to fresh serum-free media containing the indicated concentrations of apamin $(0.5,1$ and $2 \mu \mathrm{g} / \mathrm{ml})$. After $24 \mathrm{~h}$, the cells were replaced with fresh serum-free media containing $2 \mathrm{ng} / \mathrm{ml}$ of TGF- $\beta 1$ for $24 \mathrm{~h}$.

Histopathology and immunohistochemistry. Hematoxylin and eosin (H\&E), Masson's trichrome and immunohistochemical staining were performed according to a previously described procedure (15). Sections were stained with H\&E and Masson's trichrome. For immunohistochemical analysis, sections were incubated with anti-fibroblast specific protein-1 (FSP-1) (ab41532; Abcam, Cambridge, UK) for $1 \mathrm{~h}$ at $37^{\circ} \mathrm{C}$, processed by an indirect immunoperoxidase technique using a commercial kit (Dako, Carpinteria, CA, USA). The slides were examined with an Eclipse 80i microscope (Nikon, Tokyo, Japan) and analyzed with iSolution DT software (IMT i-Solution, Vancouver, BC, Canada).

Immunofluorescence staining. Paraffin-embedded mouse liver sections $(3-\mu \mathrm{m}$ thickness) were prepared by a routine procedure. After blocking with $10 \%$ donkey serum for $30 \mathrm{~min}$, the slides were immunostained with primary antibodies against cytokeratin (CK)7 (ab9021), CK19 (ab52625) (Abcam), and proliferating cell nuclear antigen (PCNA) (sc-56; Santa Cruz Biotechnology, Inc., Santa Cruz, CA, USA). After washing, they were incubated with the secondary antibodies (Alexa Flour 488 and/or Alexa Fluor 594) for $30 \mathrm{~min}$ at $37^{\circ} \mathrm{C}$. Sections were then counterstained with Hoechst 33342. Stained slides were imaged using a Nikon A1+ confocal microscope (Nikon).

Immunocytochemistry. HSC-T6 cells were grown on chamber slides and were fixed with $3.7 \%$ paraformaldehyde for $10 \mathrm{~min}$. Cells were permeated with $0.5 \%$ Triton X-100 for $10 \mathrm{~min}$ and then incubated with primary antibodies against SMA (A5228; Sigma) for $1 \mathrm{~h}$ at $37^{\circ} \mathrm{C}$. After washing, the cells were incubated with the secondary antibodies (Alexa Fluor 594) for $30 \mathrm{~min}$ at $37^{\circ} \mathrm{C}$. Cells were counterstained with Hoechst 33342 . The cells were imaged using a Nikon A1+ confocal microscope.
Enzyme-linked immunosorbent assay (ELISA). Concentrations of interleukin-6 (IL-6) and interferon- $\gamma$ (IFN- $\gamma$ ) in serum were measured with ELISA kit (R\&D Systems). The OD was measured at $450 \mathrm{~nm}$ in an ELISA reader (BMG Labtech, Mornington, Germany).

Western blot analysis. Western blotting was performed as previously described (16). Primary antibodies used in this study were the following; anti-p-Smad2/3 (\#8828), anti-Smad2/3 (\#8685) (Cell Signaling Technology, Danvers, MA, USA), anti-IL-1 $\beta$ (sc-7884), anti-Smad4 (sc-7966), anti-glyceraldehyde 3-phosphate dehydrogenase (GAPDH; sc-25778) (Santa Cruz Biotechnology, Inc.), anti-SMA (A5228; Sigma), anti-tumor necrosis factor- $\alpha$ (TNF- $\alpha$; ab6671), anti-collagen I (ab34710) (Abcam), TGF- $\beta 1$ (MAB240-100; R\&D Systems) and anti-fibronectin (610077; BD Biosciences, San Diego, CA, USA).

Statistical analysis. The experimental results are expressed as mean \pm SE. ANOVA and paired or unpaired t-test were performed for statistical analysis as appropriate. p-value $<0.05$ was considered to indicate a statistically significant result. All experiments were performed at least three times.

\section{Results}

Apamin ameliorates liver damage and inflammatory hepatic injury. To investigate the effects of apamin treatment on liver fibrosis, a mouse model induced by DDC diet feeding was used. When challenged with the DDC diet for 4 weeks, the structure of the hepatic lobule was clear in the NC group (Fig. 1A) and there was a large amount of bile duct proliferation, accompanied by inflammatory cell infiltration in the DDC-fed group as shown by H\&E staining (Fig. 1C). In addition, the above pathological changes were reduced in the apamin-treated group (Fig. 1D) compared with these changes noted in the DDC-fed group. Masson's trichrome staining indicated collagen deposition surrounding the proliferated bile duct in the DDC-fed group (Fig. 2C). In contrast, apamin treatment resulted in diminished fibrosis and collagen deposition (Fig. 2D).

In cholangiopathies, inflammation and reactive proliferation of bile ducts are closely related with the development of biliary fibrosis (17). ELISA and western blot analyses indicated that expression levels of IL- 6 , IFN- $\gamma$, TNF- $\alpha$ and IL- $1 \beta$ were significantly higher in the DDC-fed group compared with these levels in the NC group (Fig. 3). However, apamin treatment attenuated inflammatory cytokine expression, including IL- 6 , IFN- $\gamma$, TNF- $\alpha$ and IL- $1 \beta$ compared with expression levels in the DDC-fed group. Taken together, these data confirm the anti-inflammatory and moderate antifibrotic effects of apamin on the DDC-fed mice.

Effect of apamin on BEC proliferation in DDC-fed mice. We next determined the effect of apamin on ductular reaction in the DDC-fed mice by immunofluorescence of CK19 expression. Chronic DDC feeding in mice was previously demonstrated to result in cholangitis and immune responses against BECs with the destruction of bile ducts and ductules (18). CK19 is regarded as a hallmark of bile epithelial cells (19). Immunofluorescence staining showed that CK19 
A

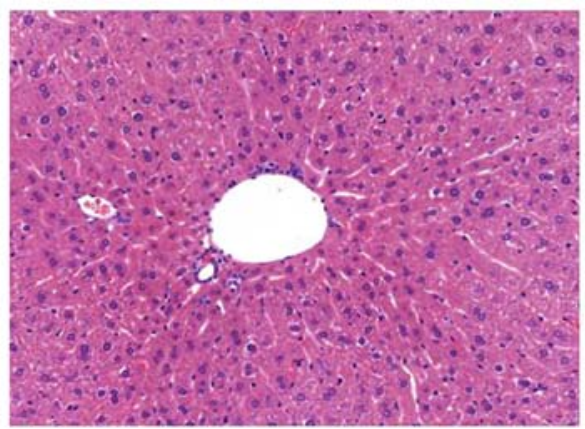

C

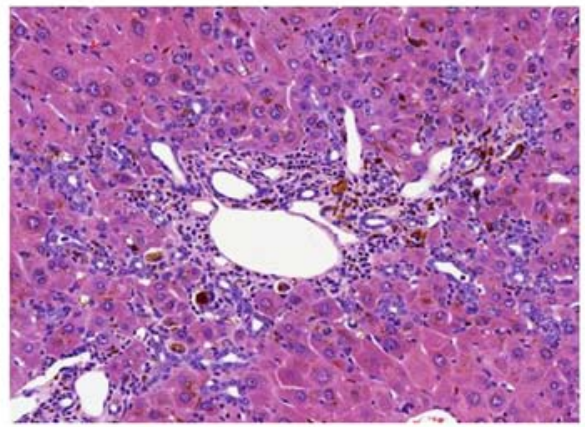

B

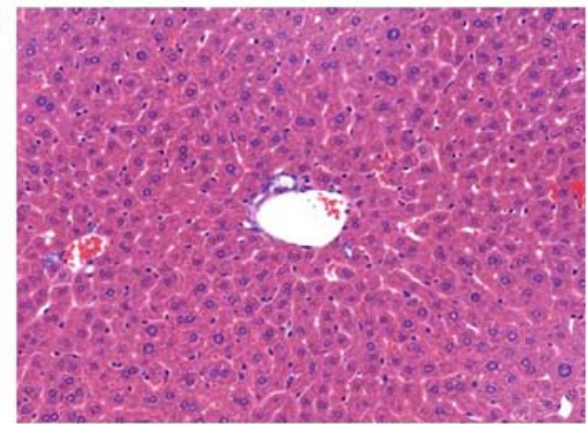

D

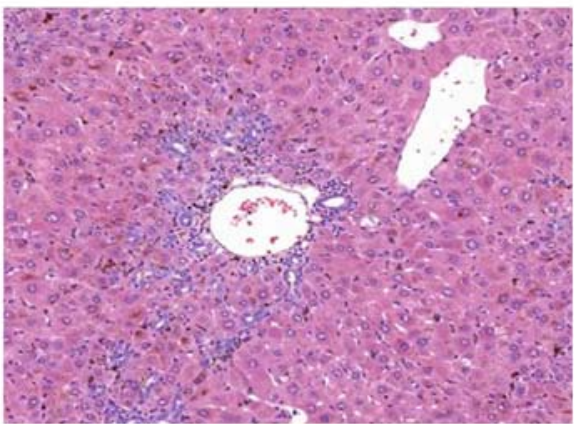

Figure 1. Apamin inhibits liver fibrosis in a 3,5-diethoxycarbonyl-1,4-dihydrocollidine (DDC)-induced mouse model as determined by hematoxylin and eosin staining. (A) Normal control, (B) apamin, (C) DDC, (D) DDC + apamin group. Histological examinations were performed at x200 magnification under light microscopy.
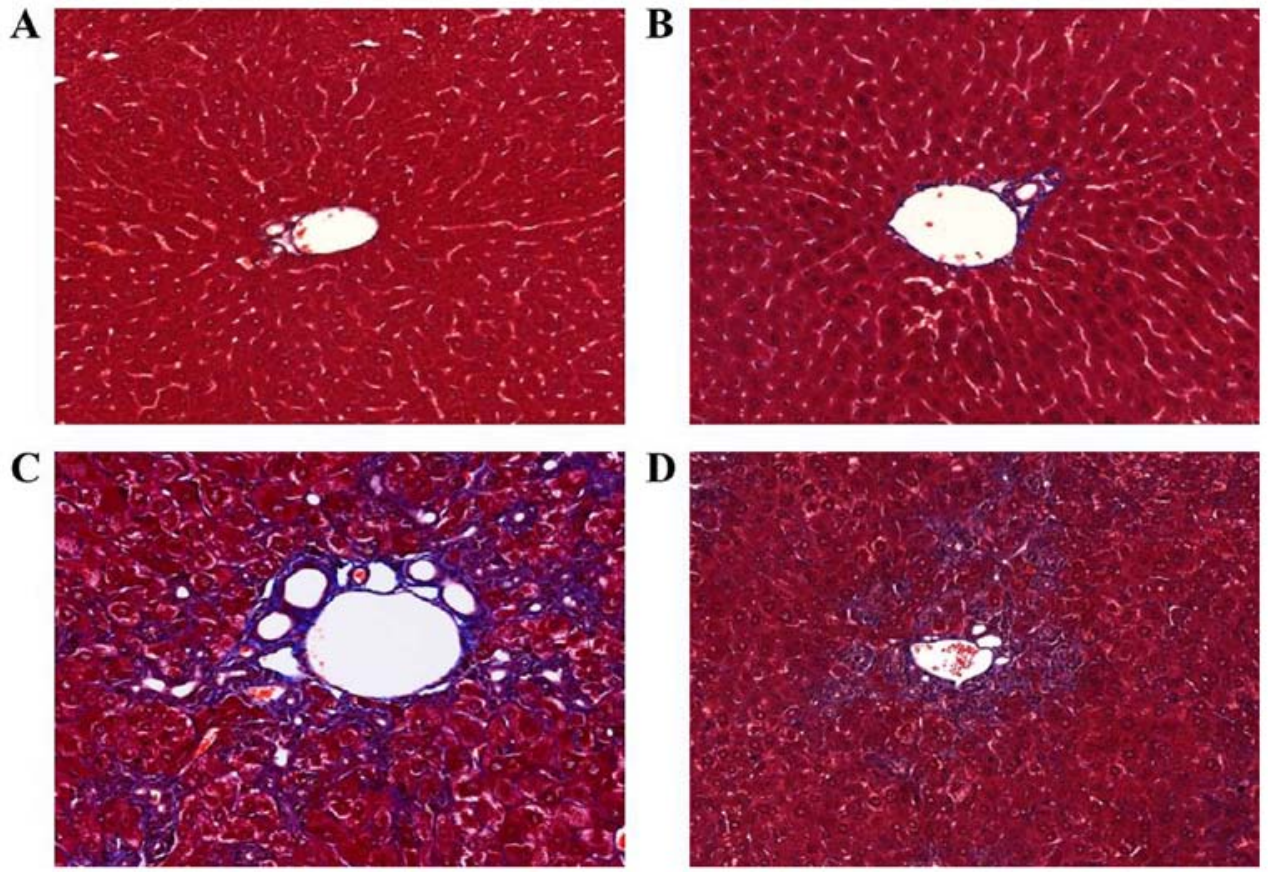

Figure 2. Apamin suppresses liver fibrosis in a 3,5-diethoxycarbonyl-1,4-dihydrocollidine (DDC)-induced mouse model as determined by Masson's trichrome staining. (A) Normal control, (B) apamin, (C) DDC, (D) DDC + apamin group. Histological examinations are performed at x200 magnification under light microscopy.

was highly expressed in the BECs in bile ductules in enlarged portal tracts (Fig. 4). The DDC-fed group had significantly increased expression of CK19 compared with the NC group. In contrast, apamin treatment significantly reduced biliary activation and proliferation as evidenced by CK19 staining, indicating a defect in the ductular reaction. In addition, immunofluorescence staining of PCNA showed that apamin treatment suppressed the proliferation of BECs compared with the DDC-fed group. These results indicate that apamin may inhibit cholestatic liver fibrosis by suppressing BEC proliferation and ductular reaction induced by the DDC diet.

Apamin inhibits ECM deposition in the livers of DDC-fed mice. To investigate the anti-fibrotic effect of apamin on ECM 

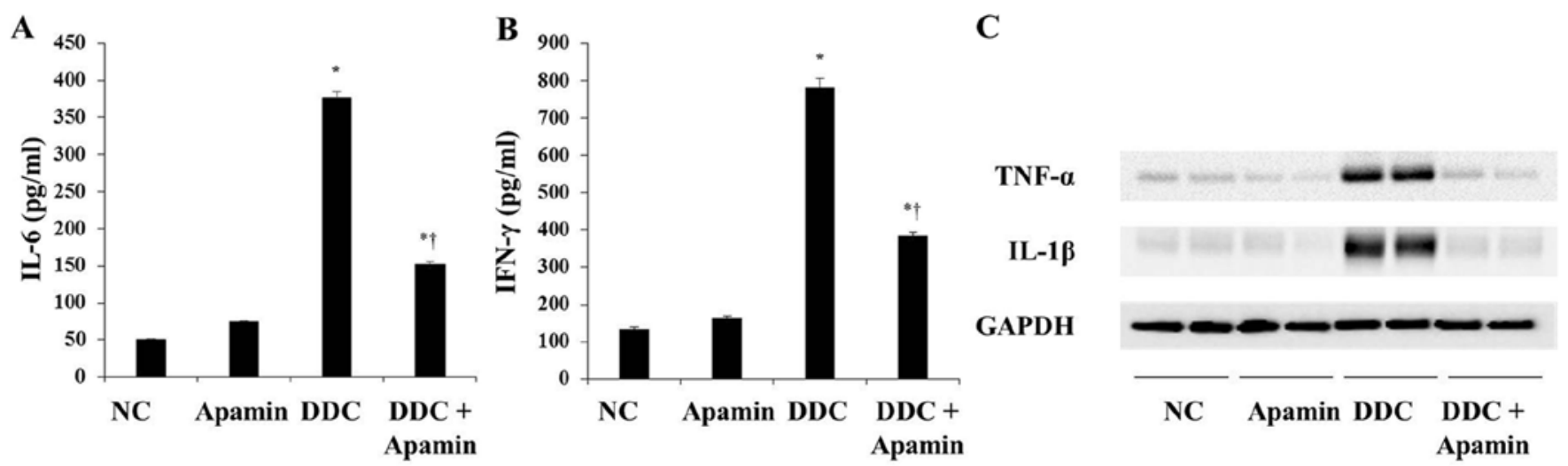

Figure 3. Apamin attenuates 3,5-diethoxycarbonyl-1,4-dihydrocollidine (DDC)-induced liver fibrosis and pro-inflammatory cytokines in mouse model Enzyme-linked immunosorbent assay (ELISA) for (A) interleukin-6 (IL-6) and (B) interferon- $\gamma$ (IFN- $\gamma$ ) expression. (C) Western blotting for tumor necrosis factor- $\alpha(\mathrm{TNF}-\alpha)$ and IL-1 $\beta$. ${ }^{*} \mathrm{p}<0.05$ vs. normal control; ${ }^{\dagger} \mathrm{p}<0.05$ vs. DDC-fed group.
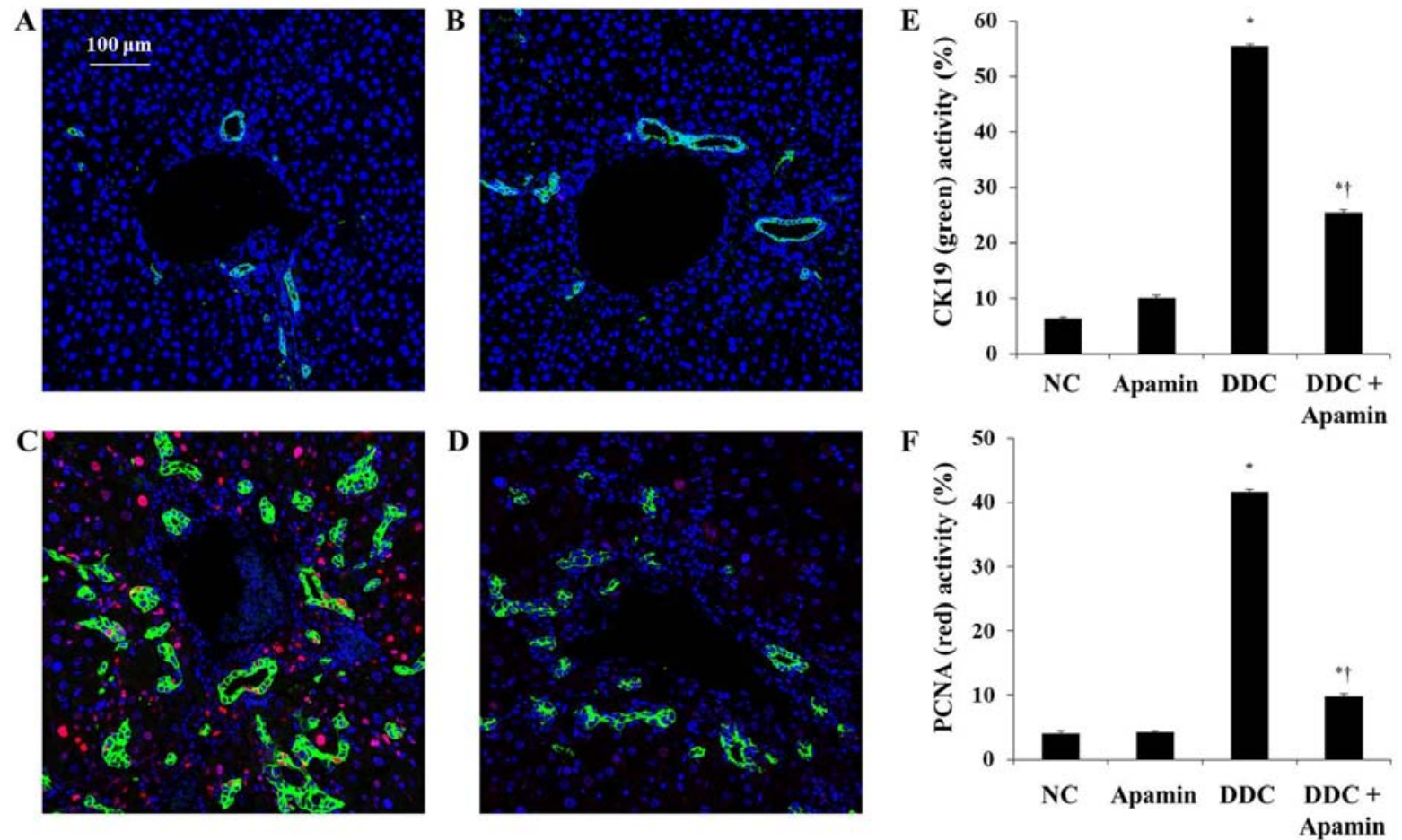

Figure 4. Apamin inhibits the proliferation of biliary epithelial cells (BECs) in a 3,5-diethoxycarbonyl-1,4-dihydrocollidine (DDC)-induced liver fibrosis mouse model. (A-D) Double immunofluorescence staining of cytokeratin 19 (CK19) (green) and proliferating cell nuclear antigen (PCNA) (red): (A) normal control, (B) apamin, (C) DDC, (D) DDC + apamin group. Scale bar, $100 \mu \mathrm{m}$. Morphometric assessment of (E) CK19- and (F) PCNA-positive areas. "p $<0.05$ vs. normal control; ${ }^{\dagger} \mathrm{p}<0.05$ vs. DDC-fed group.

deposition in the DDC-fed mice, we used western blot analysis, immunohistochemistry and immunofluorescence assay to determine the effects of this compound on ECM molecules. Liver fibrosis induced by DDC was confirmed by induction of fibrogenic genes, FSP-1, $\alpha$-smooth muscle actin ( $\alpha$-SMA) and collagen I expression. Expression of $\alpha$-SMA was strongly expressed in the myofibroblasts and HSCs around the proliferated bile duct in the DDC-fed group and clearly with the apamin treatment (Fig. 5). Moreover, expression of collagen I in the DDC-fed group was significantly increased, especially in the portal tracts. Compared to the DDC group, apamin treatment inhibited collagen I expression. During tissue remodeling in liver fibrosis, FSP-1 is considered as a marker of fibroblasts in the fibrotic liver. DDC feeding increased the number of cells positive for FSP-1 expression (Fig. 6C). In contrast, apamin treatment resulted in a reduction in FSP-1-positive cells (Fig. 6D). Furthermore, western blot results showed that the expression levels of TGF- $\beta 1$, collagen I, and fibronectin were significantly higher in the DDC-fed group, whereas apamin treatment markedly decreased the protein level of TGF- $\beta 1$, collagen I, and fibronectin compared with the DDC-fed group (Fig. 6F). Taken together, the data suggest that apamin may protect liver fibrosis during DDC feeding by suppressing fibrotic gene expression.

Apamin inhibits activation of HSCs through the Smad signaling pathway. TGF- $\beta 1$ is a Smad family member, and it 

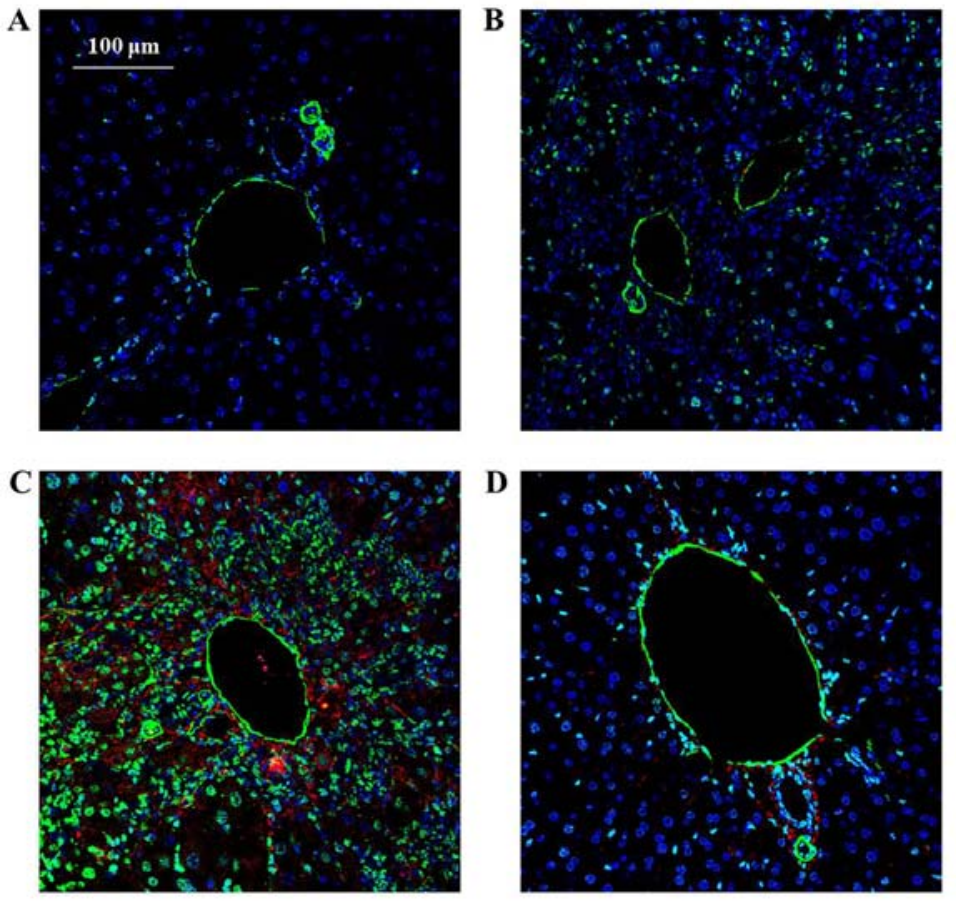
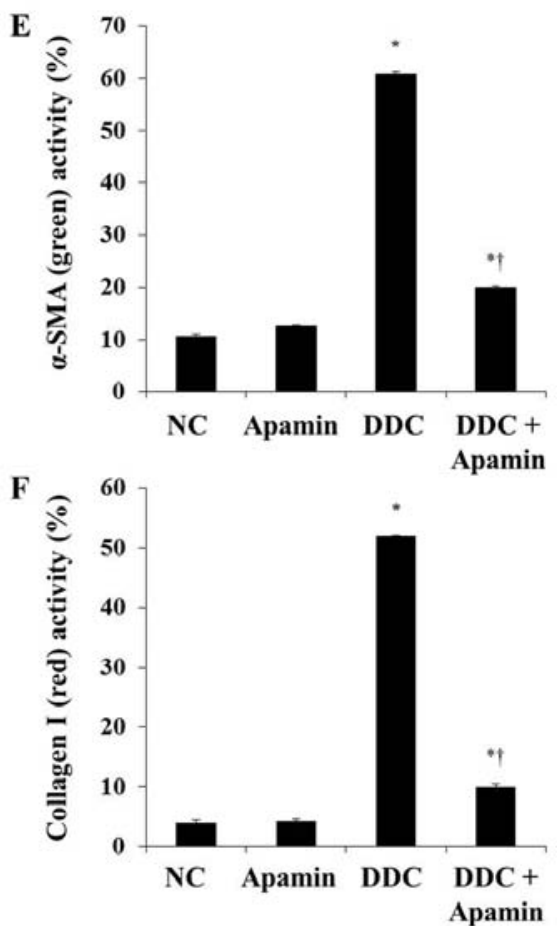

Figure 5. Apamin suppresses extracellular matrix (ECM) deposition in a 3,5-diethoxycarbonyl-1,4-dihydrocollidine (DDC)-fed liver fibrosis mouse model. (A-D) Double immunofluorescence staining of $\alpha$-smooth muscle actin ( $\alpha$-SMA) (green) and collagen I (red): (A) normal control, (B) apamin, (C) DDC, (D) DDC + apamin group. Scale bar, $100 \mu \mathrm{m}$. Morphometric assessment of (E) $\alpha$-SMA- and (F) collagen I-positive areas. " $\mathrm{p}<0.05$ vs. normal control; † $<0.05$ vs. DDC-fed group.
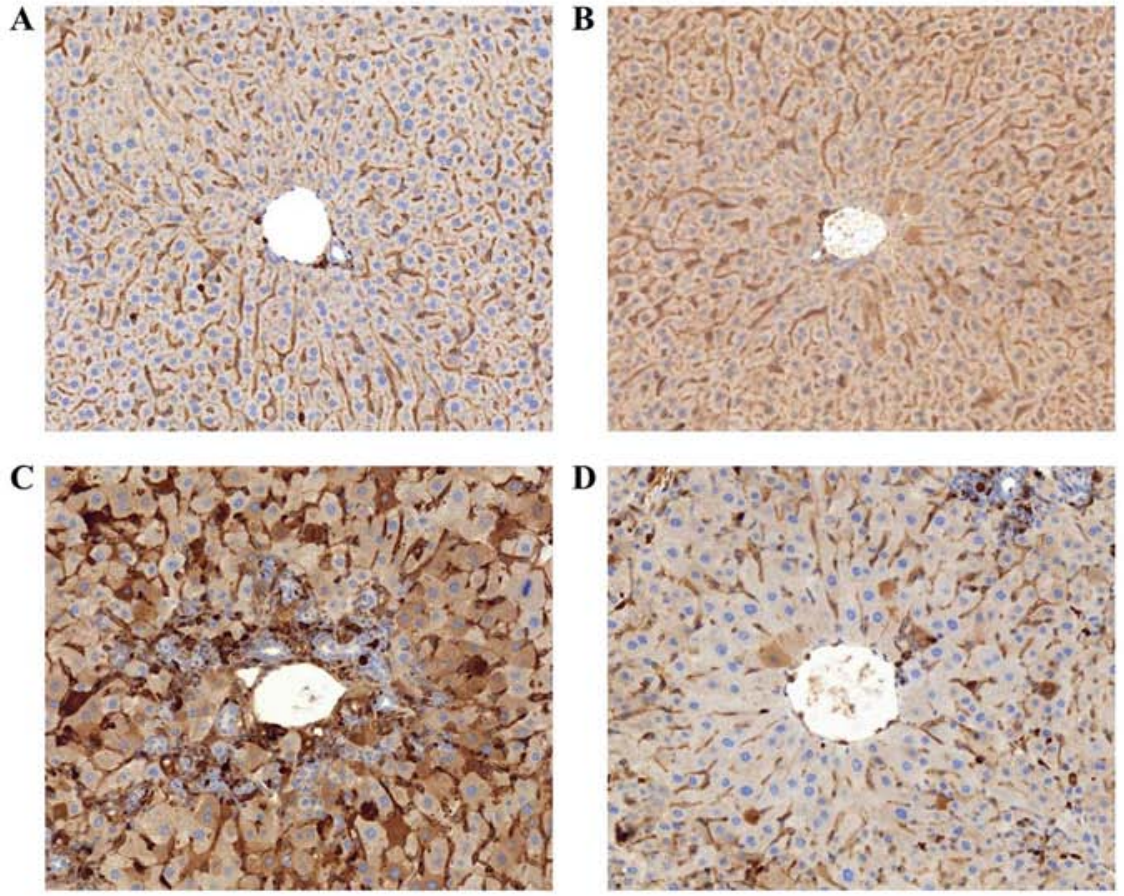

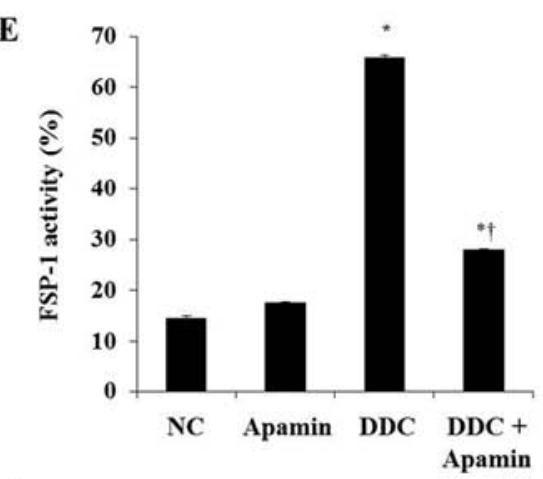

$\mathbf{F}$

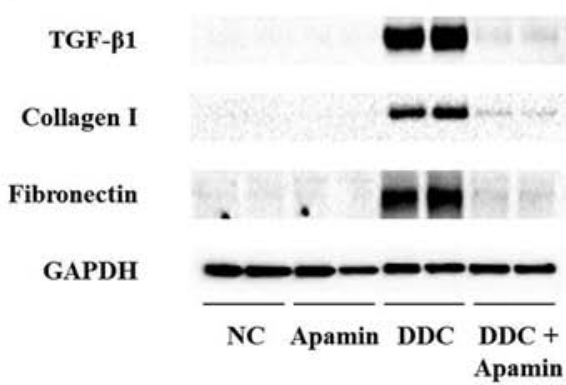

Figure 6. Apamin suppresses extracellular matrix (ECM) deposition in a 3,5-diethoxycarbonyl-1,4-dihydrocollidine (DDC)-fed liver fibrosis mouse model. (A-D) Immunohistochemical staining of fibroblast specific protein-1 (FSP-1): (A) normal control, (B) apamin, (C) DDC, (D) DDC + apamin group. Histological examinations were performed at x200 magnification under light microscopy. Morphometric assessment of (E) FSP-1-positive areas. (F) Protein expression of transforming growth factor- $\beta 1$ (TGF- $\beta 1$ ), collagen I and fibronectin. " $\mathrm{p}<0.05$ vs. normal control; ${ }^{\dagger} \mathrm{p}<0.05$ vs. DDC-fed group.

is known to stimulate the activation of HSCs (20). We next investigated whether the inhibitory effect of apamin on the activation of HSCs by TGF- $\beta 1$ is through the Smad signaling pathway. $\alpha$-SMA and collagen I expression was increased 
A

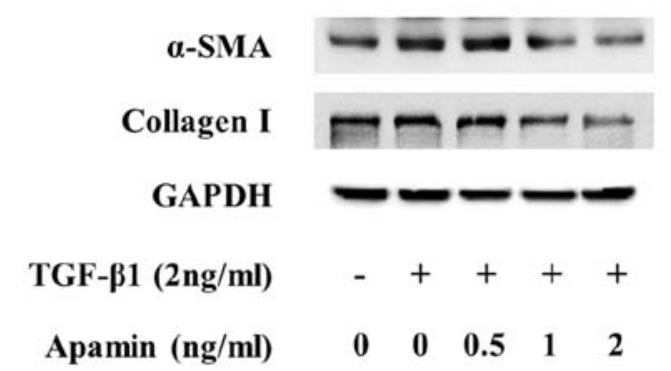

C

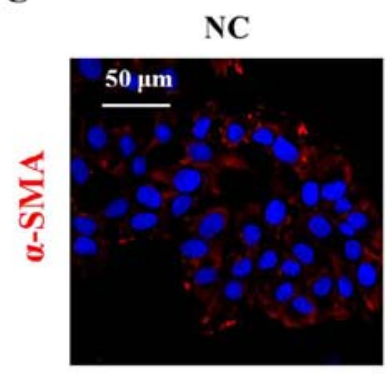

B

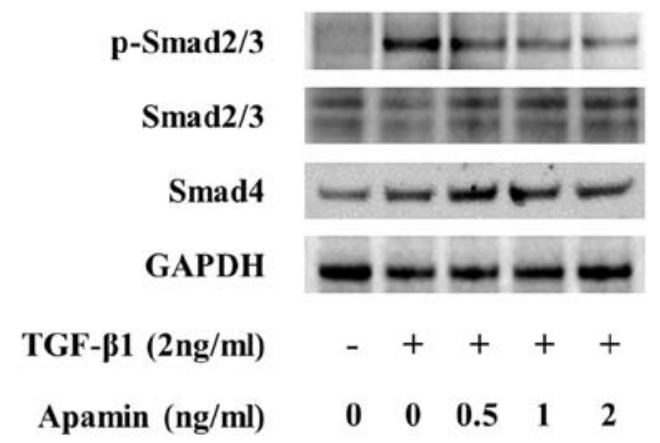
in the presence or absence of apamin for $24 \mathrm{~h}$. The cells were lysed, and proteins were analyzed by sodium dodecyl sulfate-polyacrylamide gel electrophoresis (SDS-PAGE). Western blot analysis was performed with antibodies specific for (A) $\alpha$-smooth muscle actin ( $\alpha$-SMA) and collagen I, and (B) p-Smad2/3, Smad2/3 and Smad4. Glyceraldehyde 3-phosphate dehydrogenase (GAPDH) was used as an internal control. (C) Immunocytochemistry of $\alpha$-SMA expression. Scale bar, $50 \mu \mathrm{m} .{ }^{*} \mathrm{p}<0.05$ vs. normal control; ${ }^{\dagger} \mathrm{p}<0.05$ vs. transforming growth factor- $\beta 1$ (TGF- $\beta 1$ )-treated cells.

by TGF- $\beta 1$ and was decreased by apamin treatment in the HSC-T6 cells (Fig. 7A). Also, immunofluorescence showed that TGF- $\beta 1$ induced activation of HSC-T6 cells through increased $\alpha$-SMA expression (Fig. 7C). However, apamin treatment markedly reduced the expression of $\alpha$-SMA in the TGF- $\beta 1$-induced HSC-T6 cells. Western blot analysis showed that phosphorylation of $\mathrm{Smad} 2 / 3$ and Smad4 were stimulated by $2 \mathrm{ng} / \mathrm{ml}$ TGF- $\beta 1$ (Fig. 7B). Apamin treatment abrogated the activation of $\mathrm{p}-\mathrm{Smad} 2 / 3$ and Smad 4 induced by TGF- $\beta 1$. These results indicated that apamin may attenuate TGF- $\beta 1$-activated HSCs by inhibiting the Smad signaling pathway.

\section{Discussion}

Liver fibrosis is a wound healing process that results in increased levels of ECM protein. Bee venom and its components are known to have anti-inflammatory and anti-fibrotic effects on liver fibrosis (21-24). Recent studies have shown that melittin inhibited cholangitis and biliary fibrosis in a xenobiotic-induced mouse model (15). Apamin comprises $2-3 \%$ of the dry weigh of bee venom (25). Moreover, apamin is a specific blocker of SK channels. It has been reported that apamin-sensitive $\mathrm{K}^{+}$channel is present in biliary cells and contributes to secretion in response to increased intracellular $\mathrm{Ca}^{2+}(11,26)$. Hence, we investigated the effects of apamin on the pathogenesis of DDC-induced biliary fibrosis and the suppression of activated HSCs.

The biliary system is lined by BECs, which make up $\sim 5 \%$ of all liver cells and function as important bile modi- fiers $(4,27)$. Hepatic fibrosis induced by DDC feeding is related to increased biliary pyophyrin secretion and the activation of BECs with development of bile duct injury, leading to pericholangitis and ductular reaction resulting in portal-portal fibrosis (28). Proliferating BECs can secrete a variety of profibrogenic cytokines, which promote the activation and proliferation of HSCs and also promote the synthesis of ECM, leading to the initiation and development of cholestatic hepatic fibrosis (6). Moreover, CK19 is particularly important for the proliferation of BECs during the ductular reaction, which is generally considered an attempt by the liver to restore bile flow between the lobules and the terminal bile ducts (29). In the present study, apamin significantly inhibited bile duct proliferation through a decrease in CK7, CK19 and PCNA expression in the DDC-fed mice.

TGF- $\beta 1$ is a major fibrogenic mediator involved in the activation and transdifferentiation of HSCs (30). TGF- $\beta 1$ binds to the TGF- $\beta 1$ type II receptor (T $\beta$ RII) and phosphorylates $\mathrm{Smad} 2 / 3$, and then $\mathrm{p}-\mathrm{Smad} 2 / 3$ interacts with $\mathrm{Smad} 4$ to trans-activate target genes in the nucleus (31). Smad activation is critical for the induction of many TGF- $\beta$-responsive genes including collagen I, fibronectin and $\alpha$-SMA (32). In the present study, TGF- $\beta 1$, collagen I, fibronectin and $\alpha$-SMA levels were significantly increased in the DDC-fed mouse group as determined by western blot analysis and immunofluorescence (Figs. 5 and 6F). Treatment with apamin resulted in a significant reduction in TGF- $\beta 1$, collagen $\mathrm{I}$, fibronectin and $\alpha$-SMA levels. Moreover, apamin treatment also inhibited $\alpha$-SMA expression through the Smad signaling pathway in 
the TGF- $\beta 1$-induced HSCs (Fig. 7). These findings suggest that the HSCs are transformed into myofibroblasts and secrete TGF- $\beta 1$, thus stimulating the production of ECM in the DDC-fed mouse model (15).

The principal finding of this study is the anti-fibrotic effects of apamin. Apamin suppressed the proliferation of BECs and activation of HSCs. In the present study, apamin significantly inhibited bile duct proliferation and reduced ECM deposition in the DDC-fed mice. Furthermore, apamin suppressed the protein expression of $\mathrm{p}-\mathrm{Smad} 2 / 3$ and Smad4 induced by TGF- $\beta 1$ in the HSCs. These results suggest that apamin inhibits the proliferation of BECs and activation of HSCs by suppressing the TGF- $\beta 1$ signaling pathway in hepatic fibrosis.

\section{Acknowledgements}

This study was supported by the National Research Foundation of Korea grant funded by the Korean Government (no. NRF-2015R1D1A1A01061026).

\section{References}

1. Friedman SL: Mechanisms of hepatic fibrogenesis. Gastroenterology 134: 1655-1669, 2008.

2. Gonzalez-Sanchez E, Firrincieli D, Housset C and Chignard N: Nuclear receptors in acute and chronic cholestasis. Dig Dis 33: 357-366, 2015.

3. Zollner G, Marschall HU, Wagner M and Trauner M: Role of nuclear receptors in the adaptive response to bile acids and cholestasis: Pathogenetic and therapeutic considerations. Mol Pharm 3: 231-251, 2006.

4. Glaser SS, Gaudio E, Miller T, Alvaro D and Alpini G: Cholangiocyte proliferation and liver fibrosis. Expert Rev Mol Med 11: e7, 2009.

5. Lindor KD, Gershwin ME, Poupon R, Kaplan M, Bergasa NV and Heathcote EJ; American Association for Study of Liver Diseases: Primary biliary cirrhosis. Hepatology 50: 291-308, 2009.

6. Glaser SS, Onori P, Wise C, Yang F, Marzioni M, Alvaro D, Franchitto A, Mancinelli R, Alpini G, Munshi MK, et al: Recent advances in the regulation of cholangiocyte proliferation and function during extrahepatic cholestasis. Dig Liver Dis 42: 245-252, 2010

7. Matsumoto K, Fujii H, Michalopoulos G, Fung JJ and Demetris AJ: Human biliary epithelial cells secrete and respond to cytokines and hepatocyte growth factors in vitro: Interleukin-6, hepatocyte growth factor and epidermal growth factor promote DNA synthesis in vitro. Hepatology 20: 376-382, 1994.

8. Popov Y and Schuppan D: Targeting liver fibrosis: Strategies for development and validation of antifibrotic therapies. Hepatology 50: 1294-1306, 2009.

9. Friedman SL: Molecular regulation of hepatic fibrosis, an integrated cellular response to tissue injury. J Biol Chem 275: 2247-2250, 2000.

10. Moreno M and Giralt E: Three valuable peptides from bee and wasp venoms for therapeutic and biotechnological use: Melittin, apamin and mastoparan. Toxins (Basel) 7: 1126-1150, 2015.

11. Mourre C, Fournier C and Soumireu-Mourat B: Apamin, a blocker of the calcium-activated potassium channel, induces neurodegeneration of Purkinje cells exclusively. Brain Res 778: 405-408, 1997.

12. Feranchak AP, Doctor RB, Troetsch M, Brookman K, Johnson SM and Fitz JG: Calcium-dependent regulation of secretion in biliary epithelial cells: The role of apamin-sensitive SK channels. Gastroenterology 127: 903-913, 2004.

13. Lee WR, Kim KH, An HJ, Kim JY, Lee SJ, Han SM, Pak SC and Park KK: Apamin inhibits hepatic fibrosis through suppression of transforming growth factor $\beta 1$-induced hepatocyte epithelial-mesenchymal transition. Biochem Biophys Res Commun 450: 195-201, 2014.
14. Kim SJ, Park JH, Kim KH, Lee WR, An HJ, Min BK, Han SM, Kim KS and Park KK: Apamin inhibits THP-1-derived macrophage apoptosis via mitochondria-related apoptotic pathway. Exp Mol Pathol 93: 129-134, 2012.

15. Kim KH, Sung HJ, Lee WR, An HJ, Kim JY, Pak SC, Han SM and Park KK: Effects of melittin treatment in cholangitis and biliary fibrosis in a model of xenobiotic-induced cholestasis in mice. Toxins (Basel) 7: 3372-3387, 2015.

16. Kim JY, Kim KH, Lee WR, An HJ, Lee SJ, Han SM, Lee KG, Park YY, Kim KS, Lee YS, et al: Apamin inhibits PDGF-BB-induced vascular smooth muscle cell proliferation and migration through suppressions of activated Akt and Erk signaling pathway. Vascul Pharmacol 70: 8-14, 2015.

17. Baghdasaryan A, Fuchs CD, Österreicher CH, Lemberger UJ, Halilbasic E, Påhlman I, Graffner H, Krones E, Fickert P, Wahlström A, et al: Inhibition of intestinal bile acid absorption improves cholestatic liver and bile duct injury in a mouse model of sclerosing cholangitis. J Hepatol 64: 674-681, 2016.

18. Pollheimer MJ, Fickert P and Stieger B: Chronic cholestatic liver diseases: Clues from histopathology for pathogenesis. Mol Aspects Med 37: 35-56, 2014.

19. Yongping M, Zhang X, Xuewei L, Fan W, Chen J, Zhang H, Chen G, Liu C and Liu P: Astragaloside prevents BDL-induced liver fibrosis through inhibition of notch signaling activation. J Ethnopharmacol 169: 200-209, 2015.

20. Yang JW, Hien TT, Lim SC, Jun DW, Choi HS, Yoon JH, Cho IJ and Kang KW: Pin1 induction in the fibrotic liver and its roles in TGF- $\beta 1$ expression and Smad2/3 phosphorylation. J Hepatol 60: 1235-1241, 2014

21. Kim SJ, Park JH, Kim KH, Lee WR, Chang YC, Park KK, Lee KG, Han SM, Yeo JH and Pak SC: Bee venom inhibits hepatic fibrosis through suppression of pro-fibrogenic cytokine expression. Am J Chin Med 38: 921-935, 2010.

22. Lee WR, Pak SC and Park KK: The protective effect of bee venom on fibrosis causing inflammatory diseases. Toxins (Basel) 7: 4758-4772, 2015.

23. Lee WR, Park JH, Kim KH, Park YY, Han SM and Park KK: Protective effects of melittin on transforming growth factor- $\beta 1$ injury to hepatocytes via anti-apoptotic mechanism. Toxicol Appl Pharmacol 256: 209-215, 2011.

24. Park JH, Kum YS, Lee TI, Kim SJ, Lee WR, Kim BI, Kim HS, Kim KH and Park KK: Melittin attenuates liver injury in thioacetamide-treated mice through modulating inflammation and fibrogenesis. Exp Biol Med (Maywood) 236: 1306-1313, 2011.

25. Son DJ, Lee JW, Lee YH, Song HS, Lee CK and Hong JT: Therapeutic application of anti-arthritis, pain-releasing, and anti-cancer effects of bee venom and its constituent compounds. Pharmacol Ther 115: 246-270, 2007.

26. Dutta AK, Khimji AK, Sathe M, Kresge C, Parameswara V, Esser V, Rockey DC and Feranchak AP: Identification and functional characterization of the intermediate-conductance $\mathrm{Ca}(2+)$-activated $\mathrm{K}(+)$ channel (IK-1) in biliary epithelium. Am J Physiol Gastrointest Liver Physiol 297: G1009-G1018, 2009.

27. Tabibian JH, Masyuk AI, Masyuk TV, O'Hara SP and LaRusso NF: Physiology of cholangiocytes. Compr Physiol 3: 541-565, 2013.

28. Fickert P, Stöger U, Fuchsbichler A, Moustafa T, Marschall HU, Weiglein AH, Tsybrovskyy O, Jaeschke H, Zatloukal K, Denk H, et al: A new xenobiotic-induced mouse model of sclerosing cholangitis and biliary fibrosis. Am J Pathol 171: 525-536, 2007.

29. Chen Y, Guldiken N, Spurny M, Mohammed HH, Haybaeck J, Pollheimer MJ, Fickert P, Gassler N, Jeon MK, Trautwein C, et al: Loss of keratin 19 favours the development of cholestatic liver disease through decreased ductular reaction. J Pathol 237: 343-354, 2015.

30. Leask A and Abraham DJ: TGF-beta signaling and the fibrotic response. FASEB J 18: 816-827, 2004.

31. Flanders KC: $\operatorname{Smad} 3$ as a mediator of the fibrotic response. Int $\mathbf{J}$ Exp Pathol 85: 47-64, 2004.

32. Inagaki Y and Okazaki I: Emerging insights into Transforming growth factor beta Smad signal in hepatic fibrogenesis. Gut 56: 284-292, 2007. 